\title{
Epidemiological Study and review on Chronic Insomnia; Neurological Disease
}

\section{IJCRR \\ Section: Healthcare Sci. Journal Impact Factor: 6.1 (2018) ICV: 90.90 (2018) \\ (c) (i) (8) \\ Copyright@IJCRR}

\section{Mohammad Gayoor Khan ${ }^{1}$, Thangapandi M. ${ }^{2}$, Sabitha Mary Kurian ${ }^{3}$, Veena Suresh ${ }^{4}$, D. Nagendra Babu ${ }^{4}$, Aarti Singh Kushwah' ${ }^{5}$, Umama Yezdani'}

\begin{abstract}
'Department of Pharmacology, Daksh Institute of Pharmaceutical Science, Chhatarpur, Madhya Pradesh, 471001 -lndia; 'Department of Pharmacy Practice, Karpagam College of Pharmacy, Coimbatore -21, Tamil Nadu, lndia; ${ }^{3}$ Department of Pharmacy Practice, National College of Pharmacy, Kerala India; “Department of Pharmacy Practice, Sree Datta Institute of Pharmacy Hyderabad 501510, Telangana India; ${ }^{5}$ Department of Pharmacy, Truba Institute of Pharmacy, Bhopal 462038 Madhya Pradesh India; ${ }^{6}$ Department of Pharmacy Practice, MRM College of Pharmacy, Hyderabad, Telangana, 501510 - India.
\end{abstract}

\section{ABSTRACT}

Introduction: Chronic Insomnia is a Psychological disorder or neurological disease. It is a very common condition and estimated one-fourth the population of clinical affecting by this. In short long term sleeplessness or difficulty in sleeping. In chronic insomnia case if person per week at least not sleep 3 days and this routine continues for 3-4 months regularly or longer period then the condition is considered chronic insomniac patient. Difficulty in sleeping breaks in sleepings there is several causes.

Aim: This study aims to identify particular treatment options for the treatment of insomnia.

Objective: The major objective of this study is to identify the causes of insomnia. For the treatment of the disease we first need to have a proper diagnosis after that we can provide an accurate cure to it. Various treatment measures have to be taken for the treatment of this disease including pharmacological and also non-pharmacological.

Conclusion: Treatment of this sleep disorder can be completely done. Treatment consists of modifying sleep habits to cut back involuntary and psychological feature factors and education regarding healthier sleep follow. Drug treatment ought to be reserved for the shorter alleviation of sleep disorder. Applicable treatment of chronic sleep disorder improves the standard of life. The hindrance of sleep disorder consists of a balance of rest, recreational exercise together with stress management and a healthy diet.

Key Words: Neurological disease, Chronicinsomnia, Stress, Sleep disorder.

\section{INTRODUCTION}

Insomnia is a sleep disorder which is common causes in adult women's mostly. In chronic insomnia, the patient feels difficulty in sleeping, uninterrupted sleep. ${ }^{1,2}$ Insomnia first time discovered by Johann Heinroth in 1818. It is the first psychosomatic disorder. Most of the study it is proved research adopt an arbitrary definition of insomnia as delay lower than 30 min sleep efficiency. The chronic insomnia is defined as difficulty in sleeping at least 3-4 nights in a week that repetition goes for at least 2-3 months ${ }^{3}$ or more that condition known as chronic insomnia condition.

\section{Types of insomnia}

According to NSF various types of insomnias are there. They are comorbid insomnia, acute insomnia, chronic insomnia, maintenance insomnia and onset insomnia.

Acute insomnia: It happens often and they cure without any treatment procedures. It is considered as acute insomnia when the person is unable to sleep for a few weeks. Acute insomnia happens due to stress because of job loss, loss of loved one, jet lag and divorce. Sometimes surrounding things like a baby crying, noise, light and temperature may also be the causes of this condition.

Chronic insomnia: Difficulty in sleeping over a long period. The person feels very hard to falling in asleep or continuing the sleep without interruption throughout the night in a week not less than 3 months and more. Chronic insomnia cause due to having diseases like low back pain, sleep apnea, arthritis, depression, cancer, hypothyroidism, restless

\section{Corresponding Author:}

Umama Yezdani, Department of Pharmacy Practice, MRM College of Pharmacy, Hyderabad, Telangana, 501510 - India; E-mail: umamayezdani6@gmail.com

ISSN: 2231-2196 (Print)

Received: 12.06 .2020
ISSN: $0975-5241$ (Online)

Revised: 31.07 .2020
Accepted: 23.08 .2020
Published: 22.09 .2020 
leg syndrome.

Co-Morbid insomnia: This sort of sleep deprivation happens due to some disease condition as psychiatric symptoms - anxiety and depression, cardiovascular, airway diseases, diabetes and cerebrovascular diseases.

Onset insomnia: This condition is characterized by difficulty in falling asleep at early sleeping at night.

Maintenance insomnia: This condition is described as difficulty in sleeping throughout the night. They wake up at any point of the night and have difficulty to sleep again.

Causes: Cold/ allergies, Parkinson's diseases, GERD, low back pain, sleep apnea, arthritis, depression, cancer, hypothyroidism, restless leg syndrome. Antipsychotic medication, caffeine, nicotine and biological factors such as heightened cortisol, reduced Estrogen, progesterone.

Symptoms: Dissatisfaction in sleep, day time sleeping, low energy, early wakeup, concentration difficulties, inability to do regular work, unsatisfied in between relationship, family, friends and fatigue.

Insomnia risk factors: Affects mostly women, adults more than men and older adults.Chronic illness, mental health issues and working in time.

\section{EPIDEMIOLOGY}

On an average, it is estimated that around $7-11 \%$ of the adult human population suffering from chronic insomnia which is often undertreated, under-diagnosed the condition of insomnia also increases as age passes which are most common in women's this interface with normal functioning causes anxiety, depression, disturbance increase, poor cognitive functioning mood swings and mood changes. In chronic insomnia causes slow reactions times difficulty in balance maintaining, increase risk of mortality as it results in chronic insomnia misinterpreted as mild cognitive or dementia. Every four in one person with medical condition experience insomnia disease. When compared to males women's are 1.4 times more chance to get affected by insomnia disease.About $\$ 100$ billion amount is spending every year by the people's in united states for the treatment of insomnia. Insomnia affects concentration, productivity, disability to do regular work and increases the healthcare cost of the individual who is chronically affected by insomnia. According to the 2002 NSF poll, $63 \%$ of women experience symptoms of insomnia for a few nights per week. ${ }^{4,5}$ Adults (68\%) are more affected when compared to adult ages (59\%). Surprisingly parents not having children in their household reported insomnia more when compared to parents those are having children (66 vs 54).

\section{NATURAL HISTORY}

One of the studies claimed and it is found that remission rate $13.1 \%$ after 4 months. Follow up in Population suffering from insomnia for 1 month or more than that. ${ }^{6}$

\section{PATHO PHYSIOLOGY}

Chronic insomnia is a disorder distinguished by lack of sleep and the uneasiness of falling asleep, recurrent of awakening in sleep. Chronic Insomnia has impacted $6-10 \%$ of the total adult population. Due to lack of generality, the symptoms are underrated by various age groups. Women's are mainly affected by this and it increases as age rises. This also has a rapid rate of showing up in people who suffer from diabetes. Chronic insomnia intervenes with the development of body and various systems; it convicts anxiety, emotional disorder, drowsiness, retarded psychological health. The hyperarousal creates by disturbed mind causes a higher level of cortisol and adrenocorticotropic hormone indicates towards thronging insomnia.

\section{DIAGNOSIS OF INSOMNIA}

\section{Evaluation of a patient with Insomnia}

The assessment of insomnia can be augmented by a prototype that allows for the progression of insomnia disorder.A comprehensive history retrieved during the patient interview is the linchpin of the assessment of insomnia. Whereas the interview approach may vary in consonance with the practitioner, the core should be covered to ensure an accurate evaluation. The primary objective of the evaluation process is to identify comorbid disorders that can be directly controlled. Evaluation pursues to be based on a cautious history and examination of patients dealing with sleep and waking functions, as well as prevalent medical, psychiatric, and comorbidities linked to medication/substances.

\section{Sleep History}

Components of an Insomnia intake history

\section{Principal complaints of insomnia or sleep pat- tern} Insomnia patients may complain of sleeping difficulties, recurrent awakenings, struggling to get back to sleep, early morning awakenings, or sleep that fails to feel calm, reviving, or remedial. 


\section{Key elements include:}

i. Distinguish of the type of ailments

ii. Frequency (occurrence per night)

iii. The severity of night discomfort and daytime symptoms

iv. Factors that fluctuate symptoms

v. Recognition of precipitants

vi. Perpetuating factors

vii. Duration (months, years, lifetime)

viii. Treatments and responses

ix. Course (progressive, irregular, persistent)

\section{1) Pre-sleep conditions with behavioural factors}

- Classification of the sleeping status

- The psychological state of the patient (drowsy /conscious, easy/uneasy) is useful in grasping which factors may ease or endure sleep or awakening after sleep.

\section{2) Sleep-wake schedule}

- The sleep schedule should be reviewed to comprise sack time, sleep inactivity, frequency, and duration of night time arouse, sleep re-initiation time, wake time, bedtime, and entire sleep time.

- The ability of the patient to fall asleep can be impeded by the consuming and work up times in close temporal link with bedtime. Collateral reports from relatives, teachers, or companions may evince valuable if the patient is heedless of the extent of symptoms.

\section{3) Nocturnal symptoms}

To point out nocturnal signs associated with:

- Breathing anomalies (sleep apnea)

- REM sleep behaviour disorder (kicking, punching, shouting)

- Parasomnias (abnormal movements, emotions)

- Neurological disorders/comorbid conditions (palpitations, epilepsy, headaches).

- Other Psychological emotions associated with wakefulness (restlessness, anxiety, frustration) may impart to insomnia.

\section{4) Day time activities and day time functions}

Daytime activities and behaviours can give clues about possible provocation and the outcome of insomnia such as

- $\quad$ Nod off (frequency per day, time)

- work (working hours, type of work)

- Lifestyle (inactive/active, shut-in, daytime exposure, exercise)

- travel, daytime dysfunction (cognitive dysfunction, mood, quality of life $)^{7}$

\section{5) Evaluation of Comorbid conditions}

A major part of the patient interview should be aimed at the assessment of medical conditions, mental conditions, and other sleep disorders often comorbid with insomnia. ${ }^{8}$

\section{6) Current or past treatment response}

- Over-the-counter medication approaches, alcohol, and prescription drugs must be included in the assessment of current and past treatments.

- The type, dosage, use frequency, administration time, a typical response must be evaluated

- It is also important to assess the acceptance of pharmacological and non-pharmacological therapies by the patient since these perspectives are likely to impact adherence to guidance and yield treatment outcomes. ${ }^{8}$

\section{7) Examination by physically and mentally}

- Physical examination may disclose signs compatible with sleep apnea, respiratory, thyroid, cardiac, and neurological disorders. ${ }^{9,10}$

- The mental examination may give details concerning the effect, mood, level of observant, and ability of the patient to attend. ${ }^{10}$

Table 1: Psychiatric Rating Scale of Sleep

Methods Process

Insomnia Severity Index • short, a self-administered (ISI) questionnaire which assesses the insomnia severity, sleep satisfaction, daytime impairment, perception of other's sleep problem, and patient's level of concern."

Beck Depression Inven- • BDI (BDI-II) is self -reporting tory inventory to measure the depression severity in psychiatric populations.

Epworth Sleepiness Scale

- simple, short, self-administered scale increases the chance of a dose in certain situations and therefore provides information on the daytime sleepiness of the patient, which may be the cause or effect of insomnia at night."

Fatigue Severity Scale

- FSS is a 9 statement short questionnaire to rate the level of fatigue. ${ }^{12}$

Dysfunctional beliefs and attitudes about sleep scale

- DBAS is a 28 statement selfreport questionnaires that help to assess sleep/insomnia related cognitions $^{13}$

- A 16 item short-form is available in Hindi

Pittsburgh Sleep Qual- - The self-administered questionity Index naire assesses quality, onset, duration, effectiveness, disturbance, sleep medication use, and daytime dysfunction. It helps to identify the most affected sleeping parts. 


\begin{tabular}{lll}
$\begin{array}{l}\text { State-Trait Anxiety } \\
\text { Inventory }\end{array}$ & $\begin{array}{l}\text { STAI is a self-report inventory to } \\
\text { measure state anxiety and trait } \\
\text { anxiety. A Higher score indicates } \\
\text { greater anxiety. }\end{array}$ \\
$\begin{array}{l}\text { Short Form Health } \\
\text { Survey }\end{array}$ & $\begin{array}{l}\text { SF-36 is a self-report question- } \\
\text { naire inventory, 36 items that } \\
\text { measures the quality of life. }\end{array}$ \\
Pre-sleep arousal scale & $\begin{array}{l}\text { There are two subscales of this } \\
\text { simple self-administered scale: } \\
\text { somatic and cognitive. It helps } \\
\text { to identify patients with sleep } \\
\text { disturbances and the cognitive } \\
\text { characteristics associated with } \\
\text { sleep.1, 12 }\end{array}$ \\
\hline
\end{tabular}

\section{Objective Assessment Tools}

In the evaluation of insomnia, laboratory testing, polysomnography, and actigraphy are not regularly suggested and be suitable for individuals with certain comorbid conditions/ sleep disorders.

\section{- Sleep Diaries}

Subjective sleep diaries are often used to aid for insomnia assessment in clinical settings and outcome measures in insomnia treatment. It can help to track a patient's sleepwake routines recorded over 1 to 2 weeks. Information can be collected from the diaries including sleep-wake times, the time duration in bed, and frequency in sleepwake times.

\section{- Polysomnography}

Polysomnography is not used commonly to assess insomnia; instead, it is the patient interview that is responsible for the diagnosis. It is only used to exclude the sleep existence disorderly breathing or PLMS condition.

\section{- Actigraphy}

The actigraph is an actimetry worn on the wrist of the patient. They can measure gross motor activity for 2 weeks and record rest or activity conditions. Some advanced actigraphs can fetch information such as light exposure or patient selfreporting data. The patient is presumed to be asleep when there is no movement. The data collected from the device by connecting to a computer and downloads from the actigraph, performs an asleep and wake evaluation algorithm, and presents data in numerically and graphically.

\section{- Multiple sleep latency test}

It is the evaluation tool for measuring excessive daytime sleepiness. It is the standard tool for diagnosis in narcolepsy or idiopathic hypersomnia.

\section{- Neurobehavioral testing}

The neurobehavioral and cognitive test is used to compute insomnia-associated daytime impairment. Many have found no daytime deficits objectively measured. They have to consider sensitivity and specificity when tests conducted for the daytime insomnia. ${ }^{13}$

\section{TREATMENT OF INSOMNIA}

The incidence of sleep disturbance and the degree to which insomnia remarkably affects daytime activities decide the requirement for assessment and treatment. For acute stress disorder patients, physicians begin treatment for insomnia at the initial visit where no further assessment may be needed. However, if insomnia is severe or chronic, a detailed examination is required to detect comorbidities and associated condition, psychiatric or neurologic disorder. Treatment should initiate with nonpharmacologic treatment followed by pharmacologic treatment, in case of no improvement. ${ }^{14}$

\section{NON-PHARMACOLOGICAL TREATMENT}

\section{Sleep hygiene}

It incorporates educating the patients about lifestyle modifications like wellbeing practices (substance abuse, diet, and exercise) and environmental factors (light, sound, and temperature). ${ }^{15}$ Assess sleep hygiene with the help of specific practice scales like sleep hygiene index and awareness scales. But sleep hygiene counselling alone might be unproductive in managing chronic insomnia patients and should be utilised with different aspects of cognitive behaviour therapy. ${ }^{16}$

\section{Stimulus control therapy}

It helps to initiate a regular sleep-wake cycle and involves the restriction of maladaptive behaviour like late-night use of digital devices, reading or eating on the bed. ${ }^{15}$ Recommendations include limit the utilisation of the bedroom to sleep and lovemaking and leave the room if unable to sleep in 20 minutes.

\section{Temporal control measures}

It involves a consistent time of wakening ignoring the sleep duration and avoids sleeping during the day. ${ }^{17}$ The technique involves "early to bed and early to rise, makes a person healthy, wealthy, and wise".

\section{Sleep Restriction therapy}

It is useful for patients who invest a significant amount of time in bed attempting to fall asleep, which initially restrains the time in bed to the total sleep time (TST). Thereby, it aims to improve sleep progression by utilising sleep limitation to build a sleep drive. ${ }^{18}$ The major limitation of this therapy is excessive daytime sleepiness. 


\section{Relaxation therapies}

Relaxation therapies include various activities such as

i. Autogenic training: Self-directed relaxation training by encouraging body recognitions such as warmth and heaviness of the limbs.

ii. Imagery training: Practice of generating pleasant images in mind, which helps in relaxation and stress reduction, thereby leads to good sleep.

iii. Progressive muscle relaxation: It involves straining and loosening up of various muscle groups to reduce stress and anxiety.

iv. Visual or auditory biofeedback training: It trains the patient to control certain physiologic factors like breathing, heart rate and brain activities using visual and auditory feedback to enhance from stress, anxiety and related insomnia. ${ }^{15,17}$

v. Breathing exercise, meditation or yoga: Mindfulnessbased therapy for insomnia (MBTI) is a meditationbased initiative that helps in emotion regulation and stress reduction. ${ }^{19}$

\section{Paradoxical intention therapy}

The therapy advises the patient to stay awake to reduce the anxiety associated with the strain to sleep. ${ }^{18}$

\section{Cognitive behavioural therapy}

Cognitive Behaviour Therapy for Insomnia (CBT-I) includes various components of intervention such as behavioural (sleep hygiene, stimulus control, relaxation, and sleep limitation) and psychological approaches (e.g., psychological rebuilding). ${ }^{20}$ It helps to alter false deceptions and mentalities about sleep, for example, unreasonable desires, confusions, enhancing outcomes of restlessness. The methods comprise of reattribution training (objective setting and arranging adapting reactions), decatastrophizing (planned for adjusting anxious programmed thoughts), assessment, and shifting attention. ${ }^{14}$ Effective CBT-I can show remarkable advancement in sleep onset latency (SOL), wakefulness after sleep onset (WASO) and total sleep time (TST). ${ }^{16}$

\section{Exercise}

Excess activity and exercise training help in improving the quality of sleep by significantly reducing depression and anxiety. But, avoid exercise before bedtime. ${ }^{21}$

\section{Pharmacological Management of Insomnia:}

Pharmacological therapy can be beneficial for short-term relief of insomnia but may not be efficient for long-term use.

\section{Drugs Acting on GABA-A Receptors}

Both Benzodiazepines (BZD) and benzodiazepine receptor agonists (BzRA or non-BZD) act on the gamma-aminobutyr- ic acid (GABA) receptor sites which exert anxiolytic, muscle relaxant, sedative, and hypnotic effects hence, for quite some time been acknowledged as one of the significant treatment options for insomnia. Nonetheless, certain limitations are related to long-term utilisation of such hypnotics which include the risk of tolerance and dependence. Therefore, it is recommended that BZDs should just be utilised for a brief time of about a month to prevent the incidence of the drawbacks related to long-term use

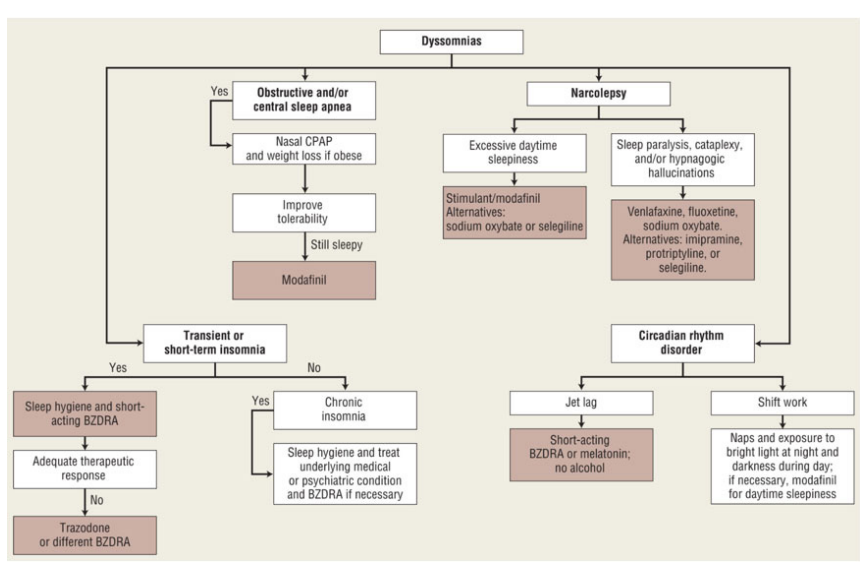

Figure 1: Algorithm for treatment of dyssomnias. (BZDRA, benzodiazepine receptor agonist; CPAP, continuous positive airway pressure. $)^{22}$

BzRA is approved by the Food and drug administration (FDA) for the treatment of insomnia. ${ }^{23}$ Compared to benzodiazepines, they are rapidly absorbed, relatively shortacting and have fewer side effects. These are useful in treating sleep maintenance insomnia, sleep onset insomnia or both.

\section{i. Zolpidem}

It binds specifically to the alpha-one subtype of GABA-A receptor. They exhibit hypnotic effects with negligible myorelaxant, anxiolytic, and anticonvulsant properties. It shows action by decreasing sleep dormancy and night-time awakenings and increases total sleep time. The side effects include falls, somnolence, headache, and antegrade amnesia.

\section{ii. Zaleplon}

Zaleplon is almost similar to Zolpidem but has the shortest span of action with a half-life of an hour. It reduces sleep latency but does not reduce nocturnal awakenings or increase total sleep time. The adverse effects include drowsiness, headache, nausea, and worsening of depression symptoms in patients associated with comorbid depressive disorder. 
Table 2: Medications Used for the Treatment of Insomnia Approved by US FDA.

\begin{tabular}{|c|c|c|c|c|}
\hline Drug & $\begin{array}{l}\text { The onset of action } \\
\text { (min) }\end{array}$ & Half-life (h) & Usual adult dose & Indications \\
\hline Estazolam* & $15-30$ & $10-24$ & $1-2$ & Sleep onset and maintenance \\
\hline Lorazepam & $30-60$ & 12 & $1-4$ & Sleep onset insomnia \\
\hline Flurazepam* & $15-20$ & $47-100$ & $15-30$ & Sleep onset and maintenance \\
\hline Quazepam* & $20-45$ & $39-73$ & $7 \cdot 5-15$ & Sleep onset and maintenance \\
\hline Temazepam* & $45-6 o$ & $3.5-8.4$ & $7 \cdot 5-15$ & Sleep onset and maintenance \\
\hline Triazolam* & $15-30$ & $1.5-5.5$ & $0.125-0.25$ & Sleep onset insomnia \\
\hline Clonazepam & $20-60$ & $30-40$ & $0.5-2$ & Sleep onset and maintenance \\
\hline Zaleplon* & 30 & 1 & $10-20$ & Sleep onset insomnia \\
\hline Zolpidem* & 30 & 2.6 & $5-10$ & Sleep onset insomnia \\
\hline Zoldipem CR* & 30 & 2.8 & $6.25-12.5$ & Sleep onset and maintenance \\
\hline Eszopiclone* $^{*}$ & 60 & 6 & $1,2,3$ & Sleep onset and maintenance \\
\hline Doxepin* & $10-20$ & $15 \cdot 3$ & $3-6$ & Sleep onset and maintenance \\
\hline Trazodone & $60-120$ & 9 & $25-100$ & Sleep onset and maintenance \\
\hline Mirtazepine & 90 & $20-40$ & $7 \cdot 5-30$ & Sleep onset and maintenance \\
\hline Agomelatine & 120 & 2 & $25-50$ & Sleep maintenance \\
\hline Hydroxyzine & $15-30$ & 13.1 & $25-50$ & Sleep onset and maintenance \\
\hline Tiagabine & $15-30$ & $7-9$ & $4-32$ & Sleep onset and maintenance \\
\hline Diphenhydramine & $6 o-18 o$ & $8-17$ & 50 & Sleep onset and maintenance \\
\hline Melatonin & $60-120$ & $3-4$ & $1-2$ & Sleep onset and maintenance \\
\hline Ramelteon* & 30 & $1-2.6$ & 8 & Sleep onset insomnia \\
\hline Suvorexant* & 30 & $10-20$ & $10-22$ & Sleep maintenance \\
\hline Quetiapine & 60 & 6 & $25-100$ & Sleep onset and maintenance \\
\hline Gabapentine & 100 & $5-9$ & $100-900$ & Sleep onset and maintenance \\
\hline
\end{tabular}

(CR: Controlled release)

iii. Eszopiclone

Eszopiclone is an isomer of zopiclone and is approved by the FDA for longer use. It aids to increase sleep productivity, daytime working alongside a decrease in sleep onset latency and wakefulness after sleep onset. The commonly observed side effects include unpleasant taste, headache, somnolence, and dizziness.

\section{Drugs Acting on Melatonin Receptors:}

The pineal gland produces a natural hormone called melatonin. The circadian system in the hypothalamus and the suprachiasmatic nucleus ( $\mathrm{SCN}$ ) regulates the levels of this hormone throughout the day and night. Melatonin is available over the counter and is approved by FDA for treatment of insomnia, especially in older adults.

\section{i. Ramelteon:}

It is a melatonin receptor agonist which decreases the sleep latency by acting on the melatonin MT1 and MT2 receptors in the SCN with higher affinity than melatonin itself. It exerts minimal adverse effects, including somnolence, fatigue, and dizziness. It should be advised with caution in pregnancy and is contraindicated in severe hepatic impairment. 
Table 3: Medications Available for Treatment of Insomnia

\begin{tabular}{|c|c|c|c|}
\hline Drug & $\begin{array}{l}\text { Brand Structure } \\
\text { Name }\end{array}$ & IUPAC Name & Mechanism of action \\
\hline Estazolam & Prosom & $\begin{array}{l}\text { 8-chloro-6-phenyl- } \\
4 \mathrm{H}-[1,2,4] \text { triazolo }[4,3-\mathrm{a}] \\
{[1,4] \text { benzodiazepine }}\end{array}$ & $\begin{array}{l}\text { It binds to the GABA receptor at } \\
\text { a site clear from the inhibitory } \\
\text { neurotransmitter GABA binding } \\
\text { site in the limbic system of the } \\
\text { CNS. This opens the chloride } \\
\text { channels allowing the flow of } \\
\text { chloride ions into the neuron, } \\
\text { thereby hyperpolarizing the } \\
\text { neuronal membrane, inhibits } \\
\text { neuronal transmission, thereby } \\
\text { decreasing neuronal excitability. }\end{array}$ \\
\hline Lorazepam & Ativan & $\begin{array}{l}\text { 7-chloro-5-(2- } \\
\text { chlorophenyl)-3-hy- } \\
\text { droxy-1,3-dihydro-1,4-ben- } \\
\text { zodiazepin-2-one }\end{array}$ & $\begin{array}{l}\text { Its action is similar to Esta- } \\
\text { zolam, inhibiting neuronal } \\
\text { transmission and decreasing the } \\
\text { neuronal excitability. }\end{array}$ \\
\hline
\end{tabular}

\section{Flurazepam Dalmane}

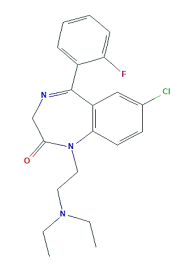

Quazepam Doral

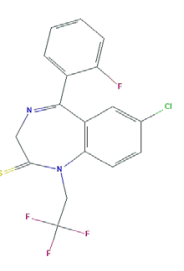

Temazepam Restoril

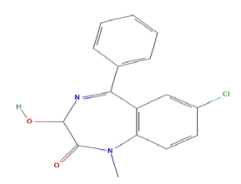

7-chloro-1-[2-

(diethylamino)ethyl]-5-(2fluorophenyl)-3H-1,4-benzodiazepine-2-one

7-chloro-5-(2-

fluorophenyl)-1-(2,2,2trifluoroethyl)-3H-1,4benzodiazepine-2-thione

7-chloro-3-hydroxy1-methyl-5-phenyl-3H-1,4benzodiazepine-2-one
It binds to a fixed site on the benzodiazepine-GABA-Achloride ionophore receptor complex located on the neuronal membrane. It creates an allosteric modification of the receptor which increases the connection of GABA to the receptor thereby, increasing the frequency of chloride-channel opening events, that leads to enhanced chloride ion transmission, leading to decreased neuronal excitability.

Quazepam and its major metabolite 2-oxoquazepam potentiate GABA to bind to BZ-1 recognition site in the GABA-A receptor, situated in the limbic, neocortical and mesencephalic reticular system. This enhances the frequency of opening of GABA-activated chloride channels, causing flow of chloride ions into the neuron, leading to decreased neuronal excitability.

Its mechanism is similar to Quazepam, enhancing the flow of chloride ions leading to decreased neuronal excitability. 
Table 3: (Continued)

Triazolam Halcion

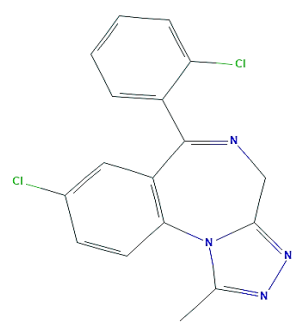

Clonazepam Klonopin

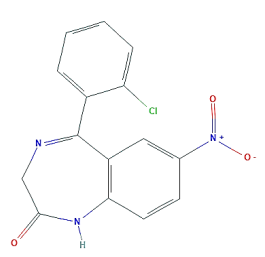

Zaleplon

Sonata

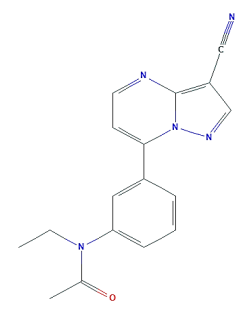

Zolpidem

Ambien, Ambien

CR,

Stilnox, and Sublinox

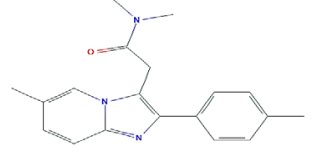

Eszopiclone Lunesta 8-chloro-6-(2chlorophenyl)-1-methyl${ }_{4} \mathrm{H}-[1,2,4]$ triazolo[4,3-a] $[1,4]$ benzodiazepine

5-(2-chlorophenyl)-7-nitro-1,3-dihydro-1,4-benzodiazepin-2-one

N-[3-(3-cyanopyrazolo[1,5a]pyrimidin-7-yl)phenyl]N-ethylacetamide

N,N-dimethyl-2-[6-methyl-2-(4-methyl phenyl) imidazo[1,2-a]pyridin-3-yl] acetamide

[(7S)-6-(5-chloropyridin2-yl)-5-oxo-7H-pyrrolo[3,4 b]pyrazin-7-yl] 4-methylpiperazine-1-carboxylate
It acts directly on a specific site on the (GABA)-A-chlorideionophore receptor complex situated on the neuronal membrane leading to allosteric changes of the receptor and variation of its affinity for the inhibitory neurotransmitter GABA, causingan increase of synaptic actions of GABA and also enhance the frequency of chloride channel openings, leading to decreased neuronal excitability.

It increases GABA receptor responses, but its mechanism of action is not completelyknown.
It actson GABA -A receptor, affecting the chloride channel ionophore complex and potentiating the inhibitory effects of GABA and also binds selectively to the brain omega-1 receptor located on the alpha subunit of the GABA-A/chloride ion channel receptor complex and potentiates t-butyl-bicyclophosphorothioate (TBPS) binding.

It initiates GABA, an inhibitory neurotransmitter, by binding to GABA-A receptors at the same location as benzodiazepines.
Its exact mechanism of action is partially known, butit binds to and activates the omega-1 subtype of the alpha subunit of the GABA-benzodiazepine GABA receptor complex (GABA-A), a chloride ionophore complex in the CNS, causing the opening of chloride channels, and increasing the inhibitory effect of GABA which leads to a hypnotic effect and allows for induction of sleep. 
Table 3: (Continued)

Doxepin Silenor

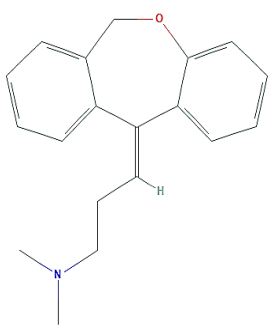

Trazodone Sideril

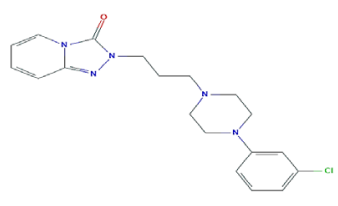

Mirtazepine Remeron, Remergil

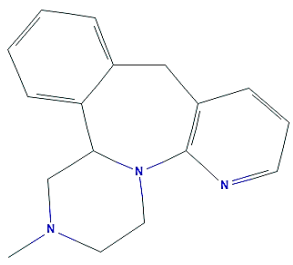

Hydroxyzine Atarax

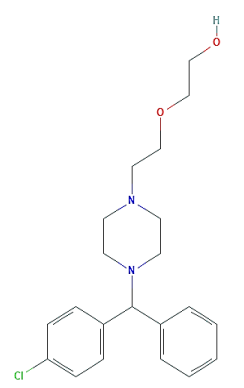

Tiagabine

Gabitril

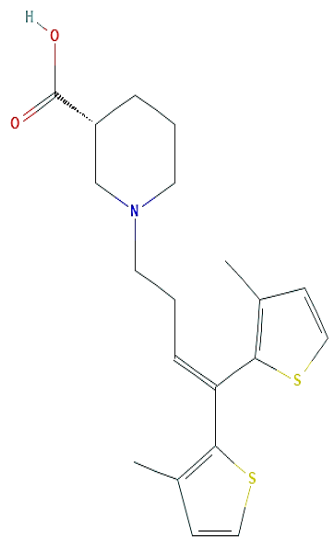

(3E)-3-(6H-benzo[c] [1] benzoxepin-11-ylidene)$\mathrm{N}, \mathrm{N}$-dimethylpropan1-amine

2-[3-[4-(3-chlorophenyl)piperazin-1-yl]propyl]-[1,2,4] triazolo[4,3-a] pyridin-3-one

5-methyl-2,5,19-triazatetracyclo[13.4.0.0 $\left.0^{2,7} \cdot 0^{8,13}\right]$ nonadeca-1(15), 8,10,12,16,18hexaene

2-[2-[4-[(4-chlorophenyl)phenylmethyl]piperazin1-yl] ethoxy] ethanol

(3R)-1-[4,4-bis(3-methylthiophen-2-yl)but-3-enyl] piperidine-3-carboxylic acid
It stops the reuptake of norepinephrine and serotonin (5-HT) into presynaptic terminals, increasing the availability of the monoaminergic neurotransmitters in the synaptic cleft and increasing its neurotransmission.

It acts as a serotonin uptake inhibitor, chemically different from other antidepressants.
Its mechanism of action is unknown, but it increases central adrenergic and serotonergic transmission, mostly by acting as an antagonist at central presynaptic alpha 2 adrenergic inhibitory autoreceptors and heteroreceptors. It is an active antagonist of 5-HT type 2 , 5-HT3, and Hi receptors, and a moderate antagonist of peripheral alpha 1 adrenergic and muscarinic receptors.

Its metabolite, Cetrizine causes the antihistaminic effect, an active $\mathrm{H}_{1}$ receptor antagonist and selective inhibitor of peripheral $\mathrm{H}_{1}$ receptors. It competes with histamine for binding at $\mathrm{H}_{1}-$ receptor sites on the effector cell surface.

Its complete mechanism is unknown, but it does appear to operate as a selective GABA reuptake inhibitor. 


\section{Table 3: (Continued)}

$\begin{array}{ll}\begin{array}{l}\text { Diphen-hy- } \\ \text { dramine }\end{array} & \text { Benadryl, } \\ \text { Genahist, } \\ \text { Naramin, } \\ \text { Sominex, } \\ \text { Unisom }\end{array}$

Melatonin Circadin

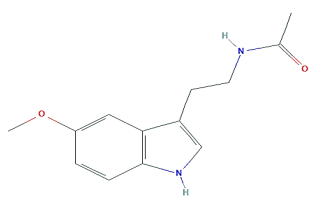

Ramelteon Rozerem

Suvorexant Belsomra

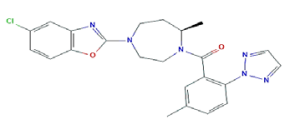

Quetiapine Seroquel

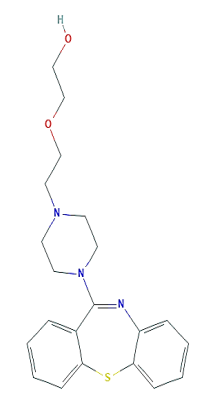

Gabapentine Neurontin
2-benzhydryloxy-N,Ndimethylethanamine

$\mathrm{N}-[2-(5-m e t h o x y-1 \mathrm{H}-\mathrm{ind}$ - 3-yl)ethyl]acetamide

$\mathrm{N}-[2-[(8 \mathrm{~S})-2,6,7,8$-tetrahydro-1H-cyclopenta[e] [1]benzofuran-8-yl]ethyl] propanamide

[(7R)-4-(5-chloro-1,3-benzoxazol-2-yl)-7-methyl-1,4diazepan-1-yl]-[5-methyl2-(triazol-2-yl)phenyl] methanone

2-[2-(4-benzo[b] [1,4]benzothiazepin-6-ylpiperazin1-yl)ethoxy] ethanol

2-[1-(aminomethyl)cyclohexyl]acetic acid
It competitively inhibits the $\mathrm{H}_{1}$ receptor, causing reducing the symptoms caused by endogenous histamine on bronchial, capillary and gastrointestinal smooth muscles.
It is a hormone produced by the pineal gland that has multiple effects including somnolence, and help in regulating the sleepwake cycle.

It is a synthetic melatonin analogue. It binds to and activates melatonin receptors 1 and 2 in the SCN of the brain, leading to the onset of sleep.

It is an orexin receptor antagonist used for the treatment of insomnia and sleep disorders.
Quetiapine fumarate antagonizes serotonin activity caused by $5_{-}$HT 1 A and 5-HT2 receptors. With a lower affinity, it also reversibly binds to dopamine D1 and D2 receptors in the mesolimbic and mesocortical areas of the brain, reducing psychotic effects, such as hallucinations and delusions. It also binds to other alpha-1, alpha-2 adrenergic and histamine $\mathrm{H}_{1}$ receptors.

It is a synthetic analogue of the neurotransmitter GABA with anticonvulsant activity. But its complete mechanism of action is unknown, it appears to inhibit excitatory neuron activity.

(GABA, gamma-aminobutyric acid; CNS, central nervous system; BZ-1, benzodiazepine-1; 5-HT, 5-hydroxytryptamine; H1, histamine 1 ; SCN, suprachiasmatic nucleus) 


\section{Antidepressants}

Doxepin acts as a pure H-1 receptor antagonist, and it exerts antihistaminic, antiserotonergic, anticholinergic and anti-adrenergic activity. It is FDA-approved for depression at doses of 100-200 mg, and insomnia at doses of 3-6 mg. Evidence from meta-analysis data reveals reduced waketime after sleep onset (WASO) and enhanced total sleep time (TST), sleep efficiency (SE) and sleep quality. The adverse effects at low doses include headache and somnolence.

${ }^{24,}{ }^{25}$ Trazodone works by inhibiting serotonin receptors (5HTA) and is widely prescribed in doses of $25-100 \mathrm{mg}$. Clinical studies show that $50 \mathrm{mg}$ once a day dose of trazodone proves to be beneficial in enhancing sleep latency, wakefulness after sleep onset and sleep duration. The common side effect observed is morning sickness. ${ }^{24}$

Tricyclic antidepressants (for example, amitriptyline, imipramine, and nortriptyline) are beneficial for inducing sleep and improving sleep quality or duration. They are an effective treatment option in patients associated with insomnia and coexisting depression. They should be advised at the lowest effective dose to decrease the chance of anticholinergic effects and cardiac conduction prolongation, particularly in geriatrics.

\section{Atypical antipsychotics}

Sedating antipsychotic drugs, such as olanzapine, quetiapine, and risperidone, exert a sedative effect at low doses mainly by their antihistaminic, anti-adrenergic and antidopaminergic properties and are effective in the treatment of insomnia with comorbid psychotic conditions. Serious adverse effects include neurological effects, weight gain, dysmetabolism and cardiometabolic effects. ${ }^{25}$

\section{Over-the-counter agents}

Antihistamines and antihistamine-analgesic combinations are generally utilised self-remedies for insomnia. Frequent use of OTC antihistamines should be prevented as they are least beneficial in inducing sleep, may reduce the quality of sleep, and can cause residual drowsiness. Diphenhydramine, doxylamine, hydroxyzine and other antihistamine drugs are commonly used to treat insomnia. These drugs also antagonise muscarinic cholinergic receptors, which can cause side effects such as urinary retention and cognitive impairment. ${ }^{25}$

\section{Alternative medications}

Valerian extracts inhibit the breakdown of $\gamma$-aminobutyric acid (GABA) or its metabolites and cause central sedation. It has little but steady effects on sleep latency, with fluctuating effects on sleep quality, duration, and design. Daytime sedation, headache and, rarely, hepatotoxicity are side effects.

\section{Prevention of insomnia:}

Lifestyle and complementary approaches that help in the prevention of insomnia include:

$>$ Regular moderate-intensity exercises, yoga, acupuncture, and acupressure relaxes mind and body, which reduces stress and thereby improves the quality of sleep. Exercising before bedtime, not recommended as it may increase alertness.

$>$ Undertaking counselling sessions for depression and PTSD might reduce the dependency on alcohol and reduce the symptoms of insomnia. ${ }^{26}$

$>$ Smoking cessation helps to reduce the occurrence of insomnia symptoms. 2Reduction of daytime naps and maintaining a consistent sleep schedule enhances the maintenance of the sleep-wake cycle.

> Light dinner 2-3 hours before bedtime and avoid foods containing caffeine, nicotine, and other stimulants, mainly in the evening time.

$>$ Relaxing activities before bedtime, such as reading, meditation, listening to music and leisure walk halfan-hour before bedtime improves the quality of sleep.

\section{CONCLUSION}

In this article, we include about chronic insomnia and morbid population able within common general population their factors related to chronic insomnia include female sex, advanced year poor general health, chronic medical disease, present or past psychiatric disorder painful conditions, social-economic status, marital status separation, poor working conditions, disturbed and social relationship. Longitudinal studies explore the dangerous factors of chronic insomnia. The treatment starts with no pharmacological intervention. Benzodiazepines and non-benzodiazepines are the major categories of drugs which are used in the treatment of insomnia. The antidepressants drugs with the antioxidant booster are very effective for the management of chronic insomnia.

Acknowledgement: All authors are thankful to Umama Yezdani, Department of Pharmacy Practice, MRM College of Pharmacy, Hyderabad Telangana India

\section{Conflict of interest: No}

Ethical clearance: Not applicable for review work in my Institute we don't use any animal/human

\section{Funding: None}

Author contribution: All authors participated equally.

\section{REFERENCES}

1. Saddichha S. Diagnosis and treatment of chronic insomnia. Ann Indian Acad Neurol 2010; 13:94-102.

2. Carney PR, Berry RB, Geyer JD. Insomnia: Causes and treat- 
ment. In: Clinical Sleep Disorders. Philadelphia, PA: Lipin Cott William and Wilkins; 2005; 157-91.

3. American Psychiatric Association. Diagnostic and Statistical Manual of Mental disorders. 5th ed. Arlington, Virginia: American Psychiatric Association; 2013.

4. Sleep disorder: symptoms and types, https://www.webmd.com/ sleep-disorders/guide/sleep-disorders-symptoms-types , cited 12.6.2020

5. Insomnio, https://www.sleepfoundation.org/sleep-disorders/insomnia, cited July 29, 2020.

6. Breslau N, Roth T, Rosenthal L, Andreski P. Sleep disturbance And psychiatric disorders: A longitudinal epidemiological study of young adults. Biol Psychiatry 1996;39:411-8.

7. Summers MO, Crisostomo MI, Stepanski EJ. Recent developments in the classification, evaluation, and treatment of insomnia. Chest. 2006;130(1):276-86.

8. Arnedt JT, Conroy DA, Posner DA, Aloia MS. Evaluation of the insomnia patient. Sleep Medicine Clinics. 2006;1(3):319-3.

9. Mai E, Buysse DJ. Insomnia: Prevalence, impact, pathogenesis, differential diagnosis, and evaluation. Focus. 2009;7(4):491-8.

10. Mai E, Buysse DJ. Insomnia: prevalence, impact, pathogenesis, differential diagnosis, and evaluation. Sleep medicine clinics. $2008 ; 3(2): 167-74$.

11. Meyer TJ. Evaluation and management of insomnia. Hospital Practice. 1998; 15;33(12):75-86.

12. Krupp LB, LaRocca NG, Muir-Nash J, Steinberg AD. The fatigue severity scale: application to patients with multiple sclerosis and systemic lupus erythematosus. Archives of neurology. $1989 ; 46(10): 1121-3$.

13. Arnedt JT, Conroy DA, Posner DA, Aloia MS. Evaluation of the insomnia patient. Sleep Medicine Clinics. 2006;1(3):319-334.

14. Buford UJ, Nemeroff CB. Current treatment options for insomnia. Drugs of Today. 2012;48(6):415-23.

15. Phore S, Panchal RS. Chronic Insomnia: A Review Suprakash. Suprakash Chaudhury, Rakesh Kumar Singh , Dolly Kumari, Chetan Diwan [Internet]. 2019;12(3):193-9.
16. Bollu PC, Kaur H. Sleep Medicine: Insomnia and Sleep. Mo Med [Internet]. 2019;116(1):68-75.

17. Maness DL, Khan M. Nonpharmacologic management of chronic insomnia. Am Fam Physician. 2015;92(12):1058-64.

18. Schutte-Rodin SL, Broch L, Buysee D, Dorsey C, Sateia M. Clinical guideline for the evaluation and management of chronic insomnia in adults. J Clin Sleep Med. 2008;4(5):487-504.

19. Ong J. A Mindfulness-Based Approach to the Treatment of Insomnia. J Clin Psychol [Internet]. 2011;23(1):1-7.

20. L. Sheng, M. Christopher AM. Evaluating treatment of obstructive sleep apnea comorbid with insomnia disorder using an incomplete factorial design. Physiol Behav. 2016;176(1):100-106.

21. Hartescu I, Morgan K, Stevinson CD. Increased physical activity improves sleep and mood outcomes in inactive people with insomnia: A randomised controlled trial. J Sleep Res. 2015;24(5):526-34.

22. Joseph T. DiPiro, Robert L. Talbert, Gary C. Yee, Gary R. Matzke, Barbara G. Wells LMP. Pharmacotherapy - A pathophysiologic approach. 8th Edition. Vol. 369, Journal of Petrology. The McGraw-Hill Companies, Inc.; 2011; 1245.

23. Takaesu Y, Komada Y, Asaoka S, Kagimura T, Inoue Y. Factors associated with long-term use of hypnotics among patients with chronic insomnia. PLoS One. 2014;9(11):6-7.

24. Sateia M, Buysse D, Krystal AD, Neubauer D, Heald J. Clinical practice guideline for the pharmacologic treatment of chronic insomnia in adults. J Clin Sleep Med J Clin Sleep Med. 2017;13(5):307-49.

25. Desai B, Desai A. Insomnia. Prim Care Emerg Physicians. 2016;309(7):281-91.

26. Miller MB, Dibello AM, Carey KB, Borsari B, Eric R, Studies A, et al. Insomnia severity as a mediator of the association between mental health symptoms and alcohol use in young adult veterans. 2018;221-7. 